\title{
ChemComm
}

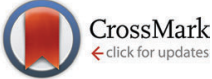

Cite this: Chem. Commun., 2017, 53, 917

Received 5th November 2016 Accepted 15th December 2016

DOI: $10.1039 / c 6 c c 08855 d$

www.rsc.org/chemcomm

\section{A sulfonated poly(arylene ether ketone)/ polyoxometalate-graphene oxide composite: a highly ion selective membrane for all vanadium redox flow batteries $\dagger$}

\author{
Md. Abdul Aziz, Kwangjin Oh and Sangaraju Shanmugam*
}

\begin{abstract}
A highly ion-selective membrane for vanadium redox flow batteries (VRBs) consisting of sulfonated poly(arylene ether ketone) (SPAEK) and polyoxometalate coupled with a graphene oxide was designed and fabricated. The SPAEK/PW-mGO composite membrane showed an effectively low self-discharge rate and excellent Coulombic efficiency $(98.73 \%)$ in VRBs.
\end{abstract}

Recent interest in large-scale energy storage technologies has increased because of the urgent requirement of grid management (peak shaving and load levelling), as a backup power or for integration and utilization of renewable energy sources. ${ }^{1-3}$ The vanadium redox flow battery (VRB) is an ideal candidate for largescale energy storage due to its high reliability, and an optimum combination of high efficiency, long cycle times, and comparatively low cost. ${ }^{4}$ However, the VRB commercial membrane Nafion exhibits several drawbacks, such as high cost (500-700 dollars per meter square $)^{5}$ and low ion-selectivity. ${ }^{6}$ Therefore, considerable attention has been paid to developing a non-fluorinated polymer membrane with high ion selectivity and adjustable ion conductivity for high-performance VRBs. ${ }^{6}$ Different non-fluorinated polymer membranes like sulfonated poly(ether ether ketone) (SPEEK), sulfonated poly(sulfone), and sulfonated poly(tetramethyldiphenyl ether ether ketone) membranes have been fabricated to reduce the cost of the membrane. ${ }^{7-9}$ The SPEEK membrane appears to be the most suitable candidate among the recently developed membranes as an alternative to the commercial Nafion membrane because of its lower cost and higher ion-selectivity. ${ }^{6,7}$ However, a high degree of sulfonation of the SPEEK membrane results in reduced mechanical strength as well as a high swelling degree that affects vanadium ion crossover through the membrane, which limits the VRB performance. ${ }^{9}$ Other alternative membranes also have less vanadium ion crossover and high Coulombic efficiency in

Department of Energy Systems Engineering, Daegu Gyeongbuk Institute of Science \& Technology (DGIST), 50-1, Sang-Ri, Hyeonpung-Myeon, Dalseong-Gun,

Daegu, 42988, Republic of Korea.E-mail: sangarajus@dgist.ac.kr

$\dagger$ Electronic supplementary information (ESI) available: Experimental details, VRB efficiency plots, and physico-chemical properties of membranes. See DOI: $10.1039 / \mathrm{c} 6 \mathrm{cc} 08855 \mathrm{~d}$
VRBs but still it is a great challenge to maintain the appropriate ratio between proton conductivity and vanadium ion permeability to achieve superior ion selectivity. ${ }^{10}$ To overcome this problem, adjusting the interfacial interaction of the composite membranes through the incorporation of fillers into the polymer matrix is a wellestablished method to overcome the conflict between proton conductivity and vanadium ion permeability. For example, inorganic compounds, such as mesoporous silica, $\mathrm{TiO}_{2}$, and graphene-based nanofillers, have been successfully incorporated into the polymer matrix to limit vanadium-ion crossover and improve mechanical strength determined by the hydrogen bonding network in the interfacial zone. .,11,12 $^{2}$

Based on this aspect, we synthesized sulfonated poly(arylene ether ketone) (SPAEK) block copolymers through nucleophilic aromatic substitution polymerization and the prepared membrane showed excellent fuel cell performance. ${ }^{13}$ In this work, we employed SPAEK as an electrolyte membrane in VRBs and compared it with a pristine Nafion membrane. Even though the pristine SPAEK membrane shows much higher VRB Coulombic efficiency than Nafion-212, the porous nature of the pristine SPAEK membrane facilitates the crossover of vanadium ions during long-term VRB operation. So, further improvement is required to explore SPAEK successfully in VRBs by modifying the SPAEK membrane to achieve high $\mathrm{H}^{+} / \mathrm{V}$ ion-selectivity. Thus, we incorporated a phosphotungstic acid (PW), $\mathrm{H}_{3}\left[\mathrm{PW}_{12} \mathrm{O}_{40}\right] 29 \mathrm{H}_{2} \mathrm{O}$ coupled graphene oxide (PW-mGO) nanohybrid into the SPAEK block copolymer to minimize the crossover of vanadium-ion species and improve the chemical stability of the obtained membrane. The PW-mGO nanohybrid was prepared based on our previous publication. ${ }^{14}$ Moreover, the polyoxometalate salts (POMs) are well-known metal-oxygen cluster compounds and have received much attention in recent years owing to their high acid strength and thermal stability. ${ }^{15}$ On the other hand, graphene oxide itself could provide a proton transport pathway via surface diffusion. ${ }^{16}$ The primary goal of introducing the PW-mGO filler into SPAEK is to provide high proton conductivity to the composite membrane as well as block the porous structure of SPAEK to reduce the vanadium-ion permeability partially by a tortuous pathway effect as depicted in Scheme 1. 
- Modified graphene oxide (mGO) Phosphotungstic acid (PW)

$\circ \mathrm{H}_{3} \mathrm{O}^{+}$

$\mathrm{V}^{\mathrm{n}^{+}}$

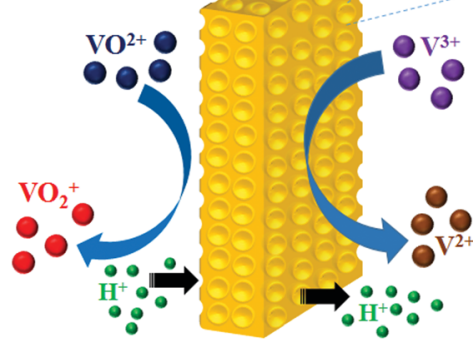

SPAEK

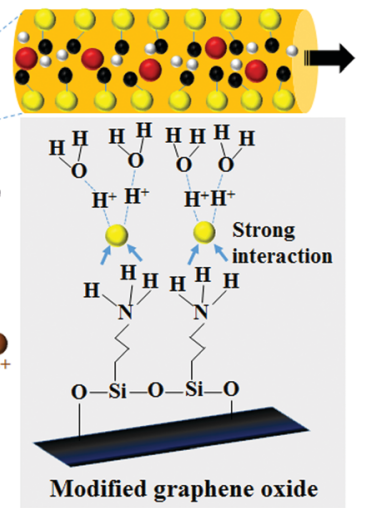

Scheme 1 Schematic illustration of VRB operation and formulation process of the SPAEK/PW-mGO composite membrane.

The SPAEK/PW-mGO composite membrane was fabricated by using a solution casting method, and the experimental details are given in the ESI. $\dagger$ The composite membranes containing PW-mGO hybrid materials of 1.0 and $2 \mathrm{wt} \%$ were denoted as SPAEK/PW-mGO (1\%) and SPAEK/PW-mGO (2\%), respectively. The SPAEK/PW-mGO membrane exhibits a homogeneous morphology, and is smooth and flexible. The dispersion of the PW-mGO filler in the SPAEK/PW-mGO composite membranes was measured using FE-SEM analysis in the backscattering mode, and it was observed that PW-mGO was randomly distributed within the membrane as shown in Fig. S1 (ESI $\dagger$ ). Moreover, upon increasing the PW-mGO filler content, the porous structure of the SPAEK membrane becomes smaller that significantly decreases vanadiumion permeability during VRB operation.

The physicochemical properties, like water uptake (WU), the swelling degree, IEC, and proton conductivity, were affected by the PW-mGO content of the membrane, and the results are listed in Table S1 (ESI $\dagger$ ). SPAEK/PW-mGO membranes showed better water uptake, swelling degree, IEC performance compared with pristine SPAEK and Nafion-212 membranes. Therefore, the proton conductivity of the composite membranes was evaluated, and the corresponding results were compared with the SPAEK and Nafion-212 membranes at room temperature under full humidity conditions as shown in Fig. 1(a). The higher proton conductivity of all the composite membranes compared to the Nafion-212 membrane was mainly due to their higher IEC values and good proton transport pathways.

In the vanadium redox flow battery (VRB), the redox species $\mathrm{V}^{2+} / \mathrm{V}^{3+}$ was used as anolyte and $\mathrm{VO}_{2}^{+} / \mathrm{VO}^{2+}$ as catholyte in the sulfuric acid solution. The main reason for the capacity loss in the battery is the spontaneous crossover of these four redox species. The nature of the redox species crossover using the same membrane is clearly different based on their different ionic diameters, and the self-discharge rate is evaluated by considering $\mathrm{VO}^{2+}$ crossover. We measured the time-dependent vanadium ion concentration in $\mathrm{MgSO}_{4}$ solution for Nafion-212, SPAEK, and SPAEK/PWmGO membranes. A linear relationship was observed between the change in vanadium ion concentration and time for the Nafion-212, SPAEK and SPAEK/PW-mGO membranes as depicted in Fig. 1(b). At a given time, the concentrations of vanadium ions crossing over through the SPAEK/PW-mGO composite membranes are lower than
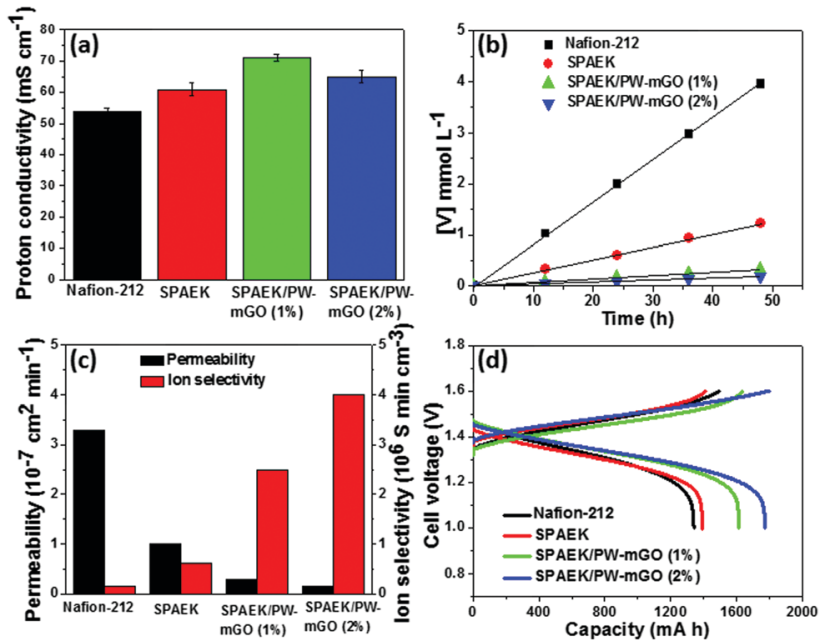

Fig. 1 (a) Proton conductivity obtained at room temperature at $100 \% \mathrm{RH}$, (b) vanadium ion $\left(\mathrm{VO}^{2+}\right)$ diffusion rate, (c) permeability of vanadium ions and ionselectivity and (d) charge-discharge curves of the VRBs assembled with Nafion212, SPAEK, SPAEK/PW-mGO (1\%) and SPAEK/PW-mGO (2\%) membranes.

that of the Nafion-212 membrane. Based on the concentration of vanadium ions crossing over, the corresponding vanadium ion permeation rate and ion-selectivity values were evaluated and are listed in Table S1 (ESI $\dagger$ ). Fig. 1(c) shows the high vanadium-ion permeability of the Nafion-212 membrane $\left(3.3 \times 10^{-7} \mathrm{~cm}^{2} \mathrm{~min}^{-1}\right)$, while the permeabilities of the SPAEK, SPAEK/PW-mGO (1\%), and SPAEK/PW-mGO (2\%) membranes are $1.0 \times 10^{-7}, 0.28 \times 10^{-7}$ and $0.16 \times 10^{-7} \mathrm{~cm}^{2} \mathrm{~min}^{-1}$, respectively. The low permeability of the SPAEK/PW-mGO composite membrane benefits from the presence of the PW-mGO hybrid material. The oxygen functional groups of PW-mGO and the sulfonic acid group of the SPAEK matrix interact with each other and act as perm-selective barriers for vanadium-ions by the tortuous pathway effect that have partly filled the porous structure of the SPAEK membrane. The ion-selectivity of the SPAEK, SPAEK/PW-mGO (1\%) and SPAEK/PW-mGO (2\%) membranes is found to be $0.61 \times 10^{6}, 2.5 \times 10^{6}$ and $4.0 \times 10^{6} \mathrm{~S} \mathrm{~min}^{-3}$, respectively. The ion selectivities of the composite membranes are much higher than the Nafion-212 membrane $(0.16 \times$ $10^{6} \mathrm{~S} \mathrm{~min} \mathrm{~cm}^{-3}$ ). Moreover, it observed that incorporating the PW-mGO nanohybrid limits the swelling behavior of the composite membrane that is firmly interrelated to its ion-selectivity. In a VRB system, a higher degree of swelling would increase the vanadium ion crossover, which was largely affected the capacity loss of the cell. Incorporation of the PW-mGO nanohybrid in SPAEK blocks the pores, thereby reducing the swelling and vanadium ions crossover properties of composite membrane.

The VRB performance of the composite membrane is evaluated using a single cell operated at room temperature with a current density of $40 \mathrm{~mA} \mathrm{~cm}^{-2}$. Fig. 1(d) shows the discharge capacity plots of the VRBs constructed with Nafion-212, SPAEK, SPAEK/PW-MGO (1\%) and SPAEK/PW-mGO (2\%) membranes and the VRBs showed discharge capacities of 1339.6, 1394.1, 1614.8 and $1774.1 \mathrm{~mA} \mathrm{~h}$, respectively. Moreover, the SPAEK/PW-mGO (1\%) and SPAEK-PW-mGO (2\%) membranes exhibited 1.2 and 1.3-times higher discharge capacity compared with the pristine Nafion-212 membrane due to the low 

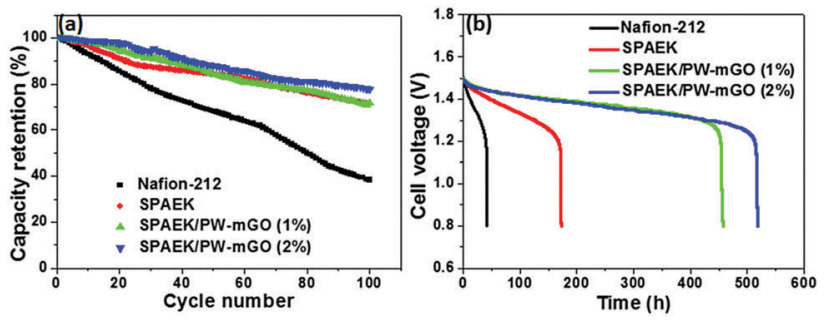

Fig. 2 (a) Discharge capacity retention of the VRB and (b) self-discharge curves of the VRBs constructed with Nafion-212, SPAEK, SPAEK/PW-mGO $(1 \%)$ and SPAEK/PW-mGO (2\%) membranes.

permeability effect of the SPAEK/PW-mGO membranes. The chargedischarge curves $\left(1-90^{\text {th }}\right.$ cycles) shown in Fig. S2 (ESI $\dagger$ ) clearly indicate the superior performance of the SPAEK/PW-mGO composite membranes. At the $90^{\text {th }}$ cycle, the charge-discharge times for the Nafion212, SPAEK, SPAEK/PW-mGO (1\%) and SPAEK/PW-mGO (2\%) membranes are 3.35, 5.84, 6.70 and $7.90 \mathrm{~h}$, respectively. Also, the SPAEK/PW-mGO (1\%) and SPAEK/PW-mGO (2\%) membranes exhibited 2 and 2.4-times higher charge-discharge times, respectively, than that of the pristine Nafion-212 membrane. The lower permeability of the SPAEK/PW-mGO membrane was reflected in a longer chargedischarge time operation. Upon increasing the charge-discharge cycle numbers, both the Nafion and composite membranes showed an increased initial IR drop. A much higher IR drop was evident for the Nafion membrane, which is due to the lower proton conductivity that increases the cell resistance, thus resulting in a faster capacity decay (Fig. 2(a)). Capacity decay during cycling is one of the vital issues for VRBs. Moreover, maintaining a stable capacity during chargedischarge is one of paramount importance. The batteries with the SPAEK, SPAEK/PW-mGO (1\%) and SPAEK/PW-mGO (2\%) membranes showed much better capacity retention compared with the Nafion-212 membrane as shown in Fig. 2(a). After 100 cycles, the capacity retention for the SPAEK, SPAEK/PW-mGO (1\%) and SPAEK/ PW-mGO (2\%) membranes is 71,72 , and $78 \%$, respectively. On the other hand, the capacity retention of the Nafion-212 membrane is only $38 \%$. The improved performance is attributed to the higher proton conductivity and less vanadium-ion crossover of the SPAEK and SPAEK/PW-mGO membranes.

Furthermore, we conducted the self-discharge test to understand the improved capacity retention of SPAEK/PW-mGO membranes. The rapid self-discharge of the VRB is due to the cross-mixing of anolyte and catholyte. The open circuit voltage (OCV) was evaluated to monitor the self-discharge rate of the VRB. Fig. 2(b) shows that the $\mathrm{OCV}$ value steadily decreased with time until $1.3 \mathrm{~V}$ and then decreases sharply to $0.8 \mathrm{~V}$ for all membranes. In contrast, the VRB assembled with a SPAEK/PW-mGO composite membrane exhibited higher performance, retaining the OCV above $1.3 \mathrm{~V}$ for the SPAEK/ PW-mGO (2\%) membrane for $441 \mathrm{~h}$, which is 3.5 and 15 -times better than that obtained with the same voltage for the SPAEK $(128 \mathrm{~h})$ and Nafion-212 (29 h) membranes, respectively. This performance is in line with the vanadium ion permeation test, in that much lower crossover of V(iv) ions occurs through the SPAEK and SPAEK/ PW-mGO membranes compared with the Nafion-212 membrane. The rapid self-discharge behavior of the SPAEK membrane compared with the SPAEK/PW-mGO composite membrane was mainly due to the sulfonated groups in polymers, which could aggregate into a hydrophilic cluster and thus facilitate a cationic transport pathway, contributing to the vanadium-ion crossover. The low vanadium ion crossover effect of the SPAEK/PW-mGO composite membranes is reflected in longer self-discharge times compared to the pristine SPAEK and Nafion-212 membranes.

The Coulombic, voltage and energy efficiencies of VRBs constructed with SPAEK and SPAEK/PW-mGO membranes were compared with a Nafion-212 membrane at $40 \mathrm{~mA} \mathrm{~cm}^{-2}$ (Fig. S3, ESI $\dagger$ ). The VRBs with the SPAEK, SPAEK/PW-mGO (1\%) and SPAEK/ PW-mGO (2\%) membranes showed Coulombic efficiencies of 97.76, 98.70 and $98.73 \%$, respectively, whereas the VRB with the Nafion-212 membrane showed much lower efficiency (89.65\%). The low Coulombic efficiency of the Nafion membrane indicates the loss of capacity attributed to the higher rate of vanadium ion crossover during charge-discharge cycles. The low permeability of the SPAEK and SPAEK/PW-mGO membranes resulted in higher Coulombic efficiency than that of the Nafion-212 membrane. Upon increasing the percentage of the $\mathrm{PW}-\mathrm{mGO}$ hybrid material, the pore size of the SPAEK membrane decreased, and resulted in lower vanadium ion crossover and a correspondingly high Coulombic efficiency of the SPAEK/PW-mGO (2\%) membrane. On the other hand, the voltage efficiency of the SPAEK, SPAEK/PW-mGO (1\%) and SPAEK/PW-mGO (2\%) membranes was $83.41,84.57$ and $82.75 \%$, respectively. The improved performance of the SPAEK/PW-mGO (1\%) membrane is due to its highest proton conductivity compared with the SPAEK/ PW-mGO (2\%) membrane. Moreover, upon increasing the percentage of the PW-mGO hybrid material, the voltage efficiency decreased gradually from 84.57 to $82.75 \%$, due to large ohmic polarization. The energy efficiency is considered to be the most important parameter in VRB performance. The SPAEK, SPAEK/PW-mGO (1\%) and SPAEK/ PW-mGO (2\%) membranes exhibited energy efficiencies of 81.56, 83.53 and $81.72 \%$, respectively, which are much greater compared to the pristine Nafion- 212 membrane $(75.53 \%)$ under the same operating conditions. The membrane thicknesses and VRB efficiencies of all the membranes are listed in Table S2 (ESI $\dagger)$. Fig. 3(a) shows different efficiencies of the SPAEK/PW-mGO (1\%) membrane, which exhibits high voltage and energy efficiencies compared with the SPAEK/PW-mGO (2\%) membrane because of its higher proton conductivity. Furthermore, upon increasing the current density from 40 to $140 \mathrm{~mA} \mathrm{~cm}{ }^{-2}$, the Coulombic efficiency is increased from $98.70 \%$ to nearly $100 \%$ for the SPAEK/PW-mGO (1\%) membrane (Fig. 3(b)). The Coulombic efficiency increased monotonically due to the shorter time for vanadium ion
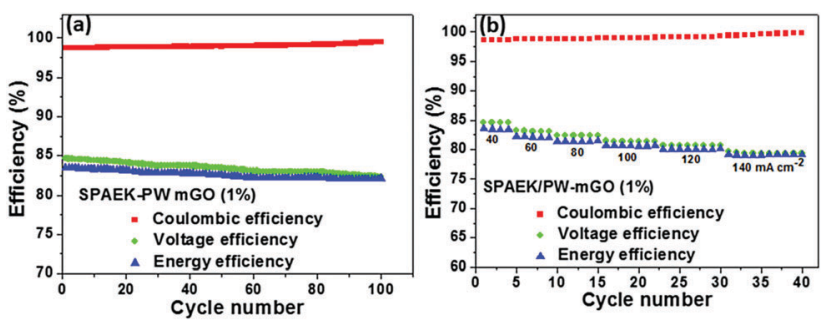

Fig. 3 Cycle efficiencies of the VRB constructed with the SPAEK/PW-mGO $(1 \%)$ membrane as a function of cycling numbers at (a) a current density of $40 \mathrm{~mA} \mathrm{~cm}^{-2}$ and (b) different current density values. 
Table 1 The performance of VRBs with SPAEK and SPAEK/PW-mGO composite membranes in comparison with previous work

\begin{tabular}{|c|c|c|c|c|c|c|c|c|}
\hline Membrane & $\begin{array}{l}\text { Thickness } \\
(\mu \mathrm{m})\end{array}$ & $\begin{array}{l}\text { Proton conductivity } \\
\left(\mathrm{mS} \mathrm{cm}^{-1}\right)\end{array}$ & $\begin{array}{l}\text { Permeability } \\
\left(\mathrm{cm}^{2} \min ^{-1}\right)\end{array}$ & $\begin{array}{l}\text { Coulombic } \\
\text { efficiency (\%) }\end{array}$ & $\begin{array}{l}\text { Energy } \\
\text { efficiency (\%) }\end{array}$ & $\begin{array}{l}\text { Current } \\
\text { density }\left(\mathrm{mA} \mathrm{cm}^{-2}\right)\end{array}$ & $\begin{array}{l}\text { Open circuit } \\
\text { voltage }(\mathrm{h})\end{array}$ & Ref. \\
\hline SPAEK/PW-MGO (1\%) & 50 & 71 & $0.28 \times 10^{-7}$ & 98.7 & 83.5 & 40 & 458 & This work \\
\hline SPAEK & 50 & 61 & $1.0 \times 10^{-7}$ & 97.8 & 81.6 & 40 & 172 & This work \\
\hline 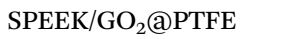 & 67 & 14.6 & $7.6 \times 10^{-7}$ & 98.4 & 81.2 & 80 & NA & 12 \\
\hline SPEEK/PAN/-20 & 55 & 15.0 & $11.3 \times 10^{-7}$ & 96.2 & 83.5 & 80 & 62 & 17 \\
\hline SPEEK & 50 & 52.0 & $3.5 \times 10^{-7}$ & 96.1 & 79.5 & 40 & NA & 18 \\
\hline AIEM-II & 45 & 48.0 & $2.9 \times 10^{-9}$ & 95.6 & 75.1 & 40 & 300 & 19 \\
\hline PVA/OMS-7 & 60 & 69.1 & NA & 97.3 & 81.5 & 30 & 68 & 20 \\
\hline Nafion-212 & 51 & 56 & $3.3 \times 10^{-7}$ & 90 & 78 & 60 & 53 & 21 \\
\hline
\end{tabular}

crossover during the charge-discharge processes at higher current density. The voltage efficiency decreases linearly from $84.62 \%$ at $40 \mathrm{~mA} \mathrm{~cm}{ }^{-2}$ to $79.33 \%$ at $140 \mathrm{~mA} \mathrm{~cm} \mathrm{~cm}^{-2}$ because of strong ohmic polarization of the cell at higher current density. The VRB using the SPAEK/PW-mGO (1\%) membrane also shows low energy efficiency loss with increasing current density. The improved performance is attributed to the high proton conductivity, low vanadium-ion crossover of these membranes and SPAEK/PW-mGO stable chemical structure.

The VRB results of the SPAEK/PW-mGO composite membrane are analyzed and compared with the other composite membranes (Table 1). The VRB assembled with the SPAEK/PW-mGO (1\%) composite membrane exhibits a lower vanadium-ion permeability $\left(0.28 \times 10^{-7} \mathrm{~cm}^{2} \mathrm{~min}^{-1}\right)$ compared with the SPEEK/graphene oxide nanocomposite $\left(\mathrm{SPEEK} / \mathrm{GO}_{2} @ \mathrm{PTFE}\right)\left(7.6 \times 10^{-7} \mathrm{~cm}^{2} \mathrm{~min}^{-1}\right){ }^{12}$ sulfonated poly(ether ether ketone)/polyacrylonitrile acid-base blend (SPEEK/PAN/-20) $\left(11.3 \times 10^{-7} \mathrm{~cm}^{2} \mathrm{~min}^{-1}\right){ }^{17}$ and sulfonated poly(ether ether ketone) (SPEEK) $\left(3.5 \times 10^{-7} \mathrm{~cm}^{2} \mathrm{~min}^{-1}\right)^{18}$ membranes. The VRB constructed with the SPAEK/PW-mGO (1\%) composite membrane showed higher Coulombic $(98.7 \%)$, voltage (84.6\%) and energy (83.5\%) efficiencies than those of the recently reported membranes as listed in Table 1 . The improved VRB performance of the SPAEK/PW-mGO (1\%) composite membrane is attributed to the low vanadium ion permeability compared with the other membranes as listed in Table 1.

The chemical stability of SPAEK/PW-mGO after 100 chargedischarge VRB operation was studied using SEM analysis. In Fig. S4 (ESI + ), the FE-SEM results indicate that the SPAEK/PW-mGO retains its original morphology without formation of any cracks or pinholes. The surface is uniform and PW-mGO fillers are nicely distributed on the surface of the membrane, which results in the excellent chemical stability of the SPAEK/PW-mGO membranes. The excellent chemical stability of the composite membrane is attributed to the strong interaction between PW-mGO and SPAEK block copolymer functional groups such as ether $(-\mathrm{O}-)$ linkage, ketone groups $(-\mathrm{C}=\mathrm{O}-)$, and sulfoxide groups $(-\mathrm{O}=\mathrm{S}=\mathrm{O}-)$. Normally, in a highly acidic environment, the ether bond of SPAEK is susceptible to the oxygen radicals, whereas in SPAEK/PW-mGO composite membrane, the filler PW-mGO can interact with the ether linkage, thereby protecting it from the oxygen radical attack under the acidic conditions.

In summary, we have successfully fabricated a composite membrane consisting of sulfonated poly(arylene ether ketone) (SPAEK) block copolymers and polyoxometalate coupled with reduced graphene oxide. The composite membrane showed improved proton conductivity $\left(71 \mathrm{mS} \mathrm{cm}^{-1}\right)$ and exhibited significantly lower vanadium-ion crossover $\left(0.28 \times 10^{-7} \mathrm{~cm}^{2} \mathrm{~min}^{-1}\right)$

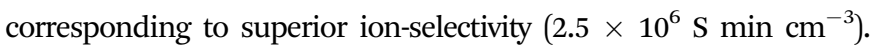
The VRB operated with the SPAEK/PW-mGO composite membrane exhibits high discharge capacity, remarkable efficiency (Coulombic efficiency, voltage efficiency and energy efficiency) in comparison with a pristine commercial Nafion-212 membrane. Due to its effectively low self-discharge rate and outstanding stability, the SPAEK/PW-mGO composite membrane is considered as a promising candidate in replacing the commercial Nafion membrane.

This work was supported by the Basic Science Research Program through the National Research Foundation of Korea (NRF) funded by the Ministry of Education, Science, and Technology (2014R1A1A2057056).

\section{Notes and references}

1 Z. Yang, J. Zhang, M. C. Kintner-Meyer, X. Lu, D. Choi, J. P. Lemmon and J. Liu, Chem. Rev., 2011, 111, 3577-3613.

2 M. Armand and J. M. Tarascon, Nature, 2008, 451, 652-657.

3 B. Dunn, H. Kamath and J. M. Tarascon, Science, 2011, 334, 928-935.

4 H. Zhang, Nature, 2014, 508, 319.

5 B. Schwenzer, J. Zhang, S. Kim, L. Li, J. Liu and Z. Yang, ChemSusChem, 2011, 4, 1388-1406.

6 X. Li, H. Zhang, Z. Mai, H. Zhang and I. Vankelecom, Energy Environ. Sci., 2011, 4, 1147-1160.

7 Z. Li, W. Dai, L. Yu, J. Xi, X. Qiu and L. Chen, J. Power Sources, 2014, 257, 221-229.

8 S. Kim, J. Yan, B. Schwenzer, J. Zhang, L. Li, J. Liu, Z. Yang and M. A. Hickner, Electrochem. Commun., 2010, 12, 1650-1653.

9 Z. Mai, H. Zhang, X. Li, C. Bi and H. Dai, J. Power Sources, 2011, 196, 482-487.

10 D. Chen, S. Wang, M. Xiao and Y. Meng, Energy Environ. Sci., 2010, 3, 622-628.

11 J. Li, Y. Zhang and L. Wang, J. Solid State Electrochem., 2014, 18, 729-737. 12 W. Dai, Y. Shen, Z. Li, L. Yu, J. Xi and X. Qiu, J. Mater. Chem. A, 2014, 2, 12423-12432.

13 K. Oh, K. Ketpang, H. Kim and S. Shanmugam, J. Membr. Sci., 2016, 507, 135-142.

14 Y. Kim, K. Ketpang, S. Jaritphun, J. S. Park and S. Shanmugam, J. Mater. Chem. A, 2015, 3, 8148-8155.

15 Y. Kim and S. Shanmugam, ACS Appl. Mater. Interfaces, 2013, 5, 12197-12204.

16 M. R. Karim, K. Hatakeyama, T. Matsui, H. Takehira, T. Taniguchi, M. Koinuma, Y. Matsumoto, T. Akutagawa, T. Nakamura, S. Noro, T. Yamada, H. Kitagawa and S. Hayam, J. Am. Chem. Soc., 2013, 135, 8097-8100.

17 Z. Li, W. Dai, L. Yu, L. Liu, J. Xi, X. Qiu and L. Chen, ACS Appl. Mater. Interfaces, 2014, 6, 18885-18893.

18 H. Y. Jung, S. Jeong and Y. Kwon, J. Electrochem. Soc., 2016, 163(1), A5090-A5096.

19 J. Qiua, M. Zhai, J. Chen, Y. Wang, J. Peng, L. Xu, J. Li and G. Wei, J. Membr. Sci., 2009, 342, 215-220.

20 S. Liu, L. Wang, B. Zhang, B. Liu, J. Wang and Y. Song, J. Mater. Chem. A, 2015, 3, 2072-2081.

21 C. Wu, S. Lu, H. Wang, X. Xu, S. Peng, Q. Tan and Y. Xiang, J. Mater. Chem. A, 2016, 4, 1174-1179. 